Conclusions ILBT can deliver high tumoricidal dose with potential improvement in the therapeutic control and better sparing of surrounding normal tissues.

\section{IDDF2019-ABS-0336 SYSTEMATIC REVIEW WITH META- ANALYSIS: THE EFFICACY OF METHOTREXATE IN ULCERATIVE COLITIS}

Shu $\mathrm{Xu}^{*}$, Hongsheng Yang, Xiuxue Hao, Yun Qiu, Shenghong Zhang, Rui Feng, Minhu Chen. Department of Gastroenterology, The First Affiliated Hospital, Sun Yat-sen University, Guangzhou, Guangdong, China

\subsection{6/gutjnl-2019-IDDFabstracts.236}

Background Although methotrexate (MTX) is used for refractory ulcerative colitis (UC) in clinical practice, there is still controversy about whether MTX is as effective in UC as that in Crohn's disease (CD).

Aim To evaluate the efficacy of MTX in UC by performing a systematic review and meta-analysis.

Methods We searched electronic databases for prospective studies evaluating the efficacy of MTX in UC. Data from cohort studies were pooled for effect estimates. Then in the meta-analysis we conducted controlled trials comparing MTX with controls.

Results Thirteen cohort studies (401 patients) were included in the meta-analysis of proportion. Mean efficacy of MTX was $26 \%$ for induction and 39\% for maintenance of remission in UC patients. Five controlled trials were included in the meta-analysis of comparison. Three studies were pooled, yielding a RR of 1.40 (95\% CI, 0.97-2.02, $P=0.07)$ for achieving clinical remission in UC patients who received MTX $(45 \%, 46 / 102)$ vs. placebo or 5 -aminosalicylic acid $(33 \%, 32 / 96)$ during the follow-up of $16-30$ weeks. Three studies were pooled, yielding a RR of 0.81 (95\% CI, 0.49-1.35, $P=0.42$ ) for maintaining clinical remission in UC patients who received MTX $(28 \%, 18 / 65)$ vs. controls $(37 \%, 22 / 60)$ during the follow-up of $32-76$ weeks. The pooled rates of severe adverse events were comparable between UC patients treated with MTX (6\%, $6 / 104)$ and placebo $(5 \%, 5 / 91$; RR 1.02 , 95\% CI $0.34-$ 3.04, $P=0.98$ ).

Conclusions Although MTX has been proved well tolerated and safe, the studies show no benefit of MTX for induction or maintenance of remission in UC patients.

\section{IDDF2019-ABS-0338 SERUM NUTRITIONAL MARKERS FOR ASSESSMENT OF PANCREATIC EXOCRINE INSUFFICIENCY IN CHRONIC PANCREATITIS PATIENTS}

Hui Xu*, Lei Liu, Bin Xu, Qi Wang, Duowu Zou. Department of Gastroenterology, Ruijin Hospital, Shanghai Jiaotong University School of Medicine, China

\subsection{6/gutjnl-2019-IDDFabstracts.237}

Background Pancreatic exocrine and endocrine insufficiency is the common complication of chronic pancreatitis (CP). Diabetes due to diseases of the exocrine pancreas was described as pancreatogenic or pan-creatogenous diabetes mellitus (DM). Available tests to assess pancreatic exocrine function in clinical practice are not widely used. The objective of this study is to investigate the potential nutritional markers for assessment of the severity of pancreatic exocrine insufficiency in CP.

Methods A retrospective analysis was performed on 158 CP patients. They were divided into two groups: CP with DM group which DM was caused by $\mathrm{CP}$ and receiving glucose-lowering treatment, and CP without DM group. Hemoglobin, albumin, prealbumin, calcium, phosphate, triglycerides (TG), total cholesterol (TC), high-density lipoprotein-cholesterol (HDL-C), low-density lipoproteincholesterol (LDL-C), apolipoprotein A, apolipoprotein B and lipoprotein (a) were analyzed and compared between these two groups.

Results Albumin, prealbumin, LDL-C were significantly lower in CP with DM group than CP without DM group (36.17 \pm 3.53 vs. $38.31 \pm 3.83, p<0.01 ; 196 \pm 60.39$ vs. 224.5 $\pm 58.07, \quad p<0.05 ; 2.11 \pm 0.68$ vs. $2.48 \pm 0.75, \quad p<0.01$; respectively). Among the 36 patients with alcoholic CP, there was no difference in serum albumin, prealbumin level between CP patients with and without DM, but LDL-C was found to be lower in the former group $(2.04 \pm 0.83$ vs. 2.63 $\pm 0.68, p<0.05)$.

Conclusions Serum nutritional markers are associated with pancreatic exocrine insufficiency and LDL-C may be used to evaluate the degree of pancreatic exocrine insufficiency in $\mathrm{CP}$.

\section{IDDF2019-ABS-0344 EXEMPLIFYING DIRECT INPATIENT COST FOR LAPAROSCOPIC MANAGEMENT OF LEFT COLONIC MALIGNANCY AT A TERTIARY CARE TEACHING HOSPITAL IN INDIA}

Suman Gupta*, Amanjeet Singh, Adarsh Chaudhary, Monish Karunakaran, Deeksha Kapoor, Nagesh Kamat. Department of GI Surgery and GI Oncology, Institute of Digestive and Hepatobiliary Sciences, Medanta -The Medicity, Gurugram, Haryana, India

\subsection{6/gutjnl-2019-IDDFabstracts.238}

Background Laparoscopic management of colorectal cancer (CRC) has been gaining popularity at a rapid pace across the world. Proponents argue for better short term outcomes including shorter hospital stay, acceptable safety profile and better outcomes of laporoscopic versus open surgery. However, the direct inpatient cost for laparoscopic management of CRC remains unexplored. This assumes even more importance in resource-strapped economies like India.

Methods A cross-sectional study was carried out with a hospital discharge diagnosis of CRC (ICD-10 C18-C19) from 2015-2018. Patient details on surgery, investigations, days of hospitalization, and drugs from hospital admission to discharge were obtained precisely from the medical records department. Item costs were obtained from the hospital electronic billing section. Economic evaluation included a prevalence-based approach with a societal perspective utilizing the bottom-up technique; cost was expressed as median cost per patient.

Results 391 (male 288; 73.6\%) patients were included. Their median age was 59 (range 49-69) years. The median time for hospitalization was 6 (range 5-11 days). The median inpatient cost was INR 1,86,564, with a wide range of $(1,69,514$ 4,51,529); [USD 2743(2492-6640); € 2332(2118-5644)]. Surgical procedure with operation theatre contributed to $30 \%$ and was the highest among all the cost components. Consumables incurred (25\%), bed charges (16\%), anesthesia (8\%), 